Article

\title{
Screening of Natural Antioxidants from Traditional Chinese Medicinal Plants Associated with Treatment of Rheumatic Disease
}

\author{
Ren-You Gan ${ }^{1}$, Lei Kuang ${ }^{1}$, Xiang-Rong Xu ${ }^{2}$, Yuan Zhang ${ }^{1}$, En-Qin Xia ${ }^{1}$, Feng-Lin Song ${ }^{1}$ \\ and Hua-Bin $\mathrm{Li}^{1, *}$
}

1 Guangdong Provincial Key Laboratory of Food, Nutrition and Health, School of Public Health, Sun Yat-Sen University, Guangzhou 510080, China; E-Mails: ganry_zsu@yahoo.cn (R.G.); kwung_1121@hotmail.com (L.K.); fly198013@yahoo.com.cn (Y.Z.); enqinxia@163.com (E.X.); sflin1986@163.com (F.S.)

2 Key Laboratory of Marine Bio-resources Sustainable Utilization, South China Sea Institute of Oceanology, Chinese Academy of Sciences, Guangzhou 510301, China;

E-Mail: xuxr2000@yahoo.com (X.X.)

* Author to whom correspondence should be addressed; E-Mail: lihuabin@mail.sysu.edu.cn; Tel.: +86-20-8733-2391; Fax: +86-20-8733-0446.

Received: 21 April 2010; in revised form: 10 August 2010 / Accepted: 13 August 2010 / Published: 30 August 2010

\begin{abstract}
In order to find new sources of natural antioxidants, the antioxidant capacities of 50 medicinal plants associated with treatment of rheumatic diseases were systemically evaluated using the ferric-reducing antioxidant power (FRAP) and Trolox equivalent antioxidant capacity (TEAC) assays, and their total phenolic contents were measured by the Folin-Ciocalteu method. Their antioxidant activities of some of these plants were analyzed for the first time. The FRAP and TEAC assay results suggested that the antioxidant compounds in these plants possessed free radicals scavenging activity and oxidant reducing power. A positive linear correlation between antioxidant capacities and total phenolic contents implied that phenolic compounds in these plants could be the main components contributing to the observed activities. The results showed that Geranium wilfordii, Loranthus parasiticus, Polygonum aviculare, Pyrrosia sheaeri, Sinomenium acutum and Tripterygium wilfordii possessed the highest antioxidant capacities and total phenolic content among 50 plants tested, and could be rich potential sources of natural antioxidants.
\end{abstract}


Keywords: medicinal plant; antioxidant capacity; total phenolic content

\section{Introduction}

Free radical-induced oxidative damage is involved in the pathogenesis of many chronic and degenerative diseases, such as cardiovascular disease, cancer, diabetes, neurodegenerative disease and ageing [1-5]. Reactive oxygen species (ROS), including superoxide free radical, hydrogen peroxide, hydroxyl free radical and singlet oxygen, play a key role in the oxidative damage of these diseases, which may result in DNA mutations, protein inactivation, lipid peroxidation, cell apoptosis or abnormal proliferation, eliciting the occurence of diseases from the cellular and molecular levels [6,7]. Antioxidants are substances capable of scavenging ROS and protecting from oxidative damage. Natural antioxidants, such as vitamins and polyphenols, have high antioxidant capacities and are abundant in many fruits and vegetables, whose consumption has been demonstrated to be inversely associated with the cardiovascular disease and some cancers [8].

The search for raw materials containing potent antioxidants continues to attract the attention of researchers. Fruit, vegetables, legume seeds and spices are all known to be rich sources of natural antioxidants, and medicinal plants are another important source for a wide variety of natural antioxidants. Recently there has been an increasing interest in natural antioxidants in Chinese medicinal plants (CMPs), and the health benefits of CMPs are thought to arise partly from the potential effects of their antioxidants on the reactive oxygen species produced in the human body. Evaluation of antioxidant activity of CMPs is very important because some plants possessing high antioxidant capacities, which are potentially valuable sources of natural antioxidants, could be screened out. In the literature, the antioxidant activities of many CMPs have been evaluated [9], and special attention has been paid to several types of CMPs, such as those possessing nutritious and tonic functions [10,11], anticancer activities [12], antiviral activities [13], heat-clearing properties [14], and blood circulation regulating actions $[15,16]$. Furthermore, several studies indicated that some CMPs possessed more potent antioxidant activities than common fruits and vegetables [12,17].

Chinese medicinal plants have been used for pharmaceutical and dietary therapy for thousands of years. In particular, there is a category of CMPs used to treat rheumatic disease, which is a series of connective tissue disorders, especially of the muscles and/or joints, pathologically characterized by inflammation, such as osteoarthritis, rheumatoid arthritis, systemic lupus erythematosus and ankylosing spondylitis. These medicinal plants have high anti-inflammatory abilities and can fight against the inflammation caused by rheumatic diseases. In addition, inflammation can be partly attributed to oxidative stress caused by ROS [18]. Furthermore, the anti-inflammatory activities of the medicinal plants could be from, at least in part, their antioxidant properties [19]. This prompted us to speculate that these medicinal plants, whose antioxidant activities were not yet evaluated systematically, could contain rich natural antioxidants. Thus, it is imperative to carry out a large-scale systematic screening of these CMPs to discover the plants with high antioxidant activities.

The purpose of this study was to systematically evaluate the antioxidant capacities and total phenolic contents of 50 Chinese medicinal plants associated with the treatment of rheumatic diseases 
in order to find new potential sources of natural antioxidants, and to investigate the relationship between total phenolic contents and antioxidant capacities. The results from this study will be in useful to understand the antioxidant capacity profiles of these medicinal plants, and also helpful for investigation of antirheumatic drugs.

\section{Results and Discussion}

\subsection{Antioxidant capacities of 50 selected medicinal plants}

The antioxidant capacities of 50 medicinal plants associated with the treatment of rheumatic diseases were evaluated using the ferric-reducing antioxidant power (FRAP) and Trolox equivalent antioxidant capacity (TEAC) assays, respectively. The FRAP assay estimates the ability to reduce ferric(III) ions to ferrous(II) ions, and the TEAC assay is used to determine the ability to scavenge $\mathrm{ABTS}^{\circ+}$ radicals, so both tests reflect the antioxidant capacities with different rationales and are simple, inexpensive, reproducible and commonly employed methods for evaluating antioxidant capacities [20-22].

Table 1. Antioxidant capacities and total phenolic contents of 50 medicinal plants.

\begin{tabular}{lccc}
\hline Scientific name & $\begin{array}{c}\text { FRAP assay } \\
(\boldsymbol{\mu m o l ~ F e ( I I ) / g ) ~}\end{array}$ & $\begin{array}{c}\text { TEAC assay } \\
(\boldsymbol{\mu m o l ~ T r o l o x / g )}\end{array}$ & $\begin{array}{c}\text { Phenolic contents } \\
(\mathbf{m g ~ G A E} / \mathbf{g})\end{array}$ \\
\hline Acanthopanax gracilistylus W. W. Smith & $170.19 \pm 3.96$ & $125.08 \pm 7.32$ & $10.23 \pm 0.20$ \\
Agadtacge rygisa O. Kuntze. & $95.19 \pm 4.53$ & $46.31 \pm 2.39$ & $4.10 \pm 0.07$ \\
Akebia trifoliata (Thunb.) Koidz. & $102.18 \pm 4.32$ & $29.15 \pm 1.29$ & $2.38 \pm 0.11$ \\
Alisma orientale (Sam.) Juz. & $5.54 \pm 0.74$ & $25.69 \pm 2.11$ & $3.90 \pm 0.16$ \\
Alpinia galanga (L.) Willd. & $82.21 \pm 2.92$ & $45.45 \pm 0.82$ & $4.25 \pm 0.10$ \\
Alpinia katsumadai Hayat & $42.89 \pm 0.95$ & $31.51 \pm 0.99$ & $2.52 \pm 0.08$ \\
Amomun kravanh Pierre ex Gagnep & $43.54 \pm 2.73$ & $25.50 \pm 0.88$ & $2.77 \pm 0.14$ \\
Amomum tsao-ko Crevostet Lemarie & $130.16 \pm 2.85$ & $100.61 \pm 1.71$ & $7.15 \pm 0.17$ \\
Amomum villosum Lour & $117.57 \pm 1.43$ & $80.16 \pm 0.97$ & $9.29 \pm 0.13$ \\
Angelica biserrata Yuan et Shan & $68.99 \pm 3.26$ & $32.67 \pm 2.03$ & $7.63 \pm 0.58$ \\
Artemisia capillaris Thunb. & $158.87 \pm 7.50$ & $106.55 \pm 3.63$ & $8.38 \pm 0.20$ \\
Atractylodes lancea (Thunb.) DC. & $28.15 \pm 0.25$ & $17.61 \pm 2.56$ & $2.41 \pm 0.41$ \\
Benincasa hispida (Thunb.) Cogn. & $57.28 \pm 4.34$ & $40.81 \pm 0.42$ & $4.21 \pm 0.21$ \\
Capsella bursapastoris (L.) Medic. & $69.99 \pm 7.85$ & $41.37 \pm 4.01$ & $4.35 \pm 0.09$ \\
Chaenomeles speciosa (Sweet) Nakai & $195.15 \pm 2.78$ & $107.61 \pm 1.09$ & $13.58 \pm 0.13$ \\
Clematis chinensis Osbeck & $82.27 \pm 4.41$ & $45.04 \pm 0.70$ & $7.85 \pm 0.03$ \\
Coix lacryma-jobi L. & $7.75 \pm 0.15$ & $4.69 \pm 0.16$ & $2.34 \pm 0.44$ \\
Cynanchum paniculatum (Bge.) Kitag. & $35.93 \pm 0.62$ & $39.45 \pm 3.81$ & $4.35 \pm 0.10$ \\
Dianthus superbus L. & $68.57 \pm 2.00$ & $37.12 \pm 3.94$ & $5.00 \pm 0.07$ \\
Dioscorea collettii Hook. F. & $16.05 \pm 0.11$ & $10.82 \pm 0.28$ & $1.31 \pm 0.03$ \\
Dioscorea nipponica Makino. & $39.64 \pm 0.51$ & $54.86 \pm 3.27$ & $4.82 \pm 0.22$ \\
Drosera burmannii Vahl & $99.80 \pm 3.70$ & $79.76 \pm 4.18$ & $4.76 \pm 0.15$ \\
Erythrina variegata L. & $185.91 \pm 0.68$ & $124.31 \pm 5.69$ & $9.12 \pm 0.23$ \\
Eupatorium fortunei Turcz. & $111.80 \pm 9.46$ & $71.93 \pm 0.55$ & $8.65 \pm 0.10$ \\
Gentiana macrophylla Pall. & $52.29 \pm 1.69$ & $27.55 \pm 1.05$ & $6.89 \pm 0.48$ \\
\hline & & & \\
& & &
\end{tabular}


Table 1. Cont.

\begin{tabular}{lccc} 
Geranium wilfordii Maxim. & $347.33 \pm 7.99$ & $215.98 \pm 4.10$ & $14.98 \pm 0.64$ \\
Homalomena occulta (Lour.) Schott & $32.30 \pm 0.47$ & $35.57 \pm 0.61$ & $3.40 \pm 0.12$ \\
Juncus effusus L. & $56.69 \pm 7.33$ & $34.77 \pm 0.94$ & $3.00 \pm 0.18$ \\
Kochia scparia (L.) Schrad & $103.22 \pm 2.28$ & $68.83 \pm 1.69$ & $8.63 \pm 0.12$ \\
Liquidambar formosana Hance & $118.44 \pm 2.44$ & $81.88 \pm 11.11$ & $5.58 \pm 0.07$ \\
Loranthus parasiticus (L.) Merr. & $580.02 \pm 31.32$ & $457.00 \pm 6.41$ & $29.67 \pm 0.99$ \\
Lysima chiachristinae Hance & $88.85 \pm 1.44$ & $65.30 \pm 0.78$ & $6.99 \pm 0.05$ \\
Magnolia officinalis Rehd. et Wils. & $257.45 \pm 9.28$ & $188.70 \pm 12.01$ & $9.68 \pm 0.22$ \\
Malva verticillata L. & $30.67 \pm 2.17$ & $20.32 \pm 0.73$ & $2.18 \pm 0.07$ \\
Morus alba L. & $46.96 \pm 3.00$ & $33.03 \pm 2.38$ & $1.96 \pm 0.33$ \\
Pinus tabulaeformis Carr. & $17.72 \pm 0.25$ & $13.30 \pm 0.26$ & $1.46 \pm 0.01$ \\
Piper kadsura (Choisy) Ohwi & $147.41 \pm 3.64$ & $103.41 \pm 8.11$ & $8.94 \pm 0.16$ \\
Plantago asiatica L. & $88.06 \pm 13.26$ & $39.94 \pm 1.41$ & $3.34 \pm 0.43$ \\
Plantago major L. & $137.23 \pm 7.07$ & $37.77 \pm 0.85$ & $6.62 \pm 0.18$ \\
Polygonum aviculare L. & $263.19 \pm 4.73$ & $171.65 \pm 10.78$ & $18.00 \pm 0.25$ \\
Polyporus umbellatus (Pers) Fr. & $7.93 \pm 1.59$ & $4.18 \pm 0.09$ & $0.38 \pm 0.05$ \\
Poria cocos (Schw.) Wolf. & $3.88 \pm 0.15$ & $1.31 \pm 0.11$ & $0.10 \pm 0.01$ \\
Pyrola calliantha H. Andr. & $160.96 \pm 5.25$ & $115.77 \pm 10.03$ & $9.31 \pm 0.72$ \\
Pyrrosia sheaeri (Bak.) Ching & $316.72 \pm 4.82$ & $147.26 \pm 4.87$ & $20.29 \pm 0.17$ \\
Siegesbeckia orientalis L. & $91.25 \pm 4.39$ & $60.23 \pm 9.49$ & $6.18 \pm 0.14$ \\
Sinomenium acutum Rehd.et Wils. & $245.94 \pm 9.25$ & $122.24 \pm 1.74$ & $16.21 \pm 0.09$ \\
Trachelospermum jasminoides Lem. & $113.22 \pm 3.29$ & $81.21 \pm 6.10$ & $9.19 \pm 0.14$ \\
Tripterygium wilfordii Hook. F. & $217.94 \pm 2.49$ & $181.98 \pm 2.87$ & $17.51 \pm 0.30$ \\
Vigna umbellata Ohwi et Ohashi & $24.74 \pm 0.80$ & $32.82 \pm 1.62$ & $3.01 \pm 0.08$ \\
Zanthoxylum nitidum (Roxb.) DC. & $198.25 \pm 14.13$ & $37.45 \pm 1.95$ & $5.44 \pm 0.16$ \\
\hline & & & \\
& & & \\
& & &
\end{tabular}

The antioxidant capacities of these medicinal plants displayed a large variance in both the FRAP and TEAC assays (Table 1). For FRAP assay, the values ranged from 3.88 to $580.02 \mu \mathrm{mol} \mathrm{Fe(II)/g} \mathrm{dry}$ weight of plant material, the median value was $90.05 \mu \mathrm{mol} \mathrm{Fe}(\mathrm{II}) / \mathrm{g}$ dry weight, the lower quartile and upper quartile of inter-quartile range were 42.08 and $159.39 \mu \mathrm{mol} \mathrm{Fe}(\mathrm{II}) / \mathrm{g}$ dry weight, respectively. Among these plants, the top ten plants with the highest antioxidant capacities were Loranthus parasiticus, Geranium wilfordii, Pyrrosia sheaeri, Polygonum aviculare, Magnolia officinalis, Sinomenium acutum, Tripterygium wilfordii, Zanthoxylum nitidum, Chaenomeles speciosa and Erythrina variegate, while Poria cocos was found to have the lowest antioxidant capacity. For the TEAC assay, the values ranged from 1.31 to $457.00 \mu \mathrm{mol}$ Trolox/g dry weight, the median value was $45.25 \mu \mathrm{mol}$ Trolox/g dry weight, the lower quartile and upper quartile of inter-quartile range were 32.38 and $104.20 \mu \mathrm{mol}$ Trolox/g dry weight, respectively. Among the plants, the top ten with the highest antioxidant capacities were Loranthus parasiticus, Geranium wilfordii, Magnolia officinalis, Tripterygium wilfordii, Polygonum aviculare, Pyrrosia sheaeri, Acanthopanax gracilistylus, Erythrina variegate, Sinomenium acutum and Pyrola calliantha, and again Poria cocos was found to have the lowest antioxidant capacity. These medicinal plants had higher antioxidant capacities than common fruits, vegetables and microalgae [12,17,22], and could be potential rich sources of natural antioxidants. 
A simple linear regression analysis was used to analyze the correlation between the FRAP values and TEAC values. The results exhibited a positive linear correlation (Figure 1). The regression equation $\mathrm{Y}=1.3306 \mathrm{X}+16.725$ was statistically significant $(\mathrm{F}=397.485, p<0.001)$, and the coefficient of determination $\mathrm{R}^{2}=0.892$, which suggested that the two methods were generally consistent for evaluating antioxidant capacities and antioxidants in these plants could not only reduce oxidants (ferric ions) but also scavenge free radicals $\left(\mathrm{ABTS}^{\circ+}\right)$.

Figure 1. Correlation between the antioxidant capacities measured by the FRAP and TEAC assays.

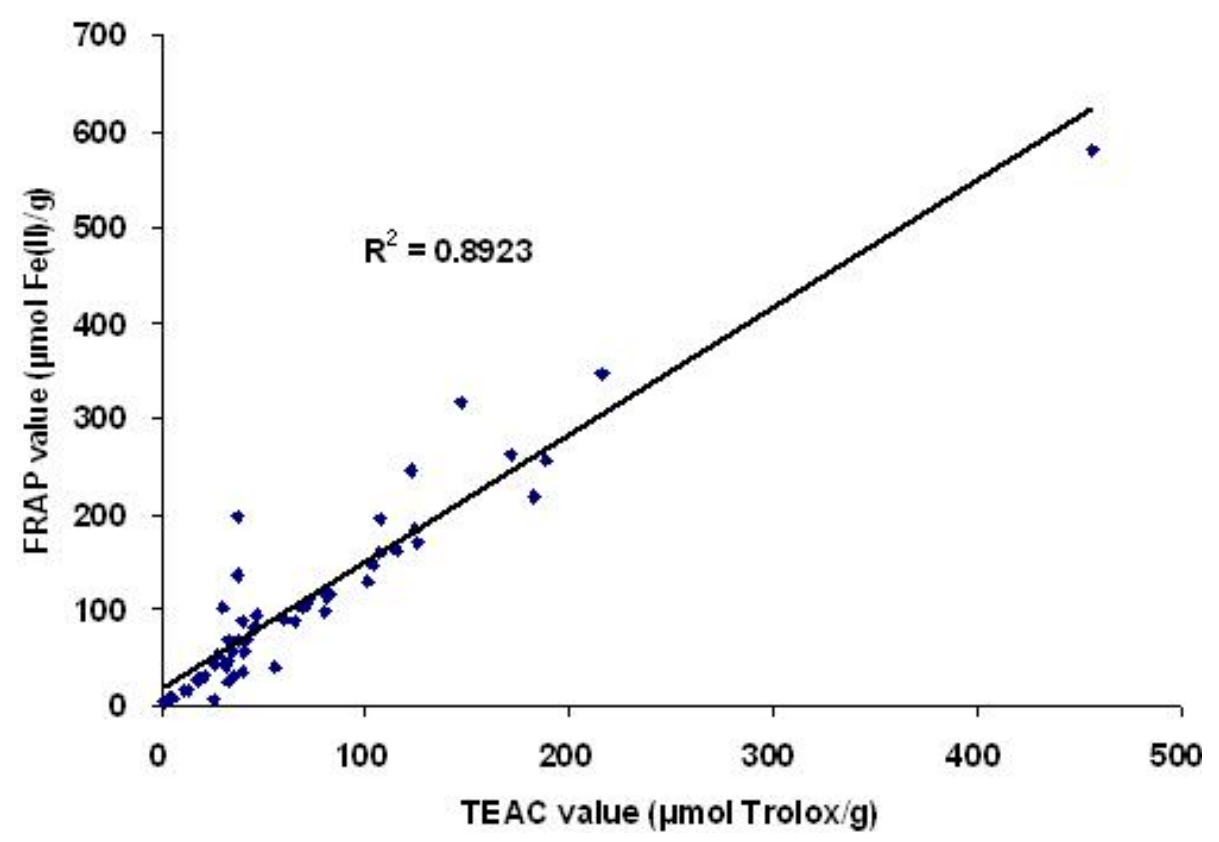

\subsection{Total phenolic content of 50 selected medicinal plants}

The Folin-Ciocalteu method was used to measure the total phenolic contents of these plants. It relies on the transfer of electrons from phenolic compounds to the Folin-Ciocalteu reagent in alkaline media [23], and is simple, reproducible and used in many studies [12,14,22,24]. Table 1 shows the total phenolic content of these medicinal plants and the values were extremely different, ranging from 0.10 to $29.67 \mathrm{mg}$ GAE$/ \mathrm{g}$ dry weight of plant material, the median value was $5.51 \mathrm{mg}$ GAE$/ \mathrm{g}$ dry weight, the lower quartile and upper quartile of inter-quartile range were 3.01 and $9.14 \mathrm{mg}$ GAE/g dry weight, respectively. Among the 50 medicinal plants, the ten plants with the highest total phenolic contents were Loranthus parasiticus, Pyrrosia sheaeri, Polygonum aviculare, Tripterygium wilfordii, Sinomenium acutum, Geranium wilfordii, Chaenomeles speciosa, Acanthopanax gracilistylus, Magnolia officinalis and Pyrola calliantha. Poria cocos had the lowest total phenolic content.

\subsection{Correlation between antioxidant capacities and total phenolic content}

Simple linear regression analysis was used to analyze the correlation between the antioxidant capacities and the total phenolic content of 50 selected medicinal plants (Figure 2). For the correlation between FRAP values and total phenolic contents, the regression equation $\mathrm{Y}=17.224 \mathrm{X}-6.7571$ was statistically significant $(\mathrm{F}=280.742, p<0.001)$, and the coefficient of determination $\mathrm{R}^{2}$ was 0.854 . 
Figure 2. Correlation between the antioxidant capacities and total phenolic content. Antioxidant capacities were measured by the FRAP assay (A) and TEAC assay (B), respectively. GAE: gallic acid equivalents.
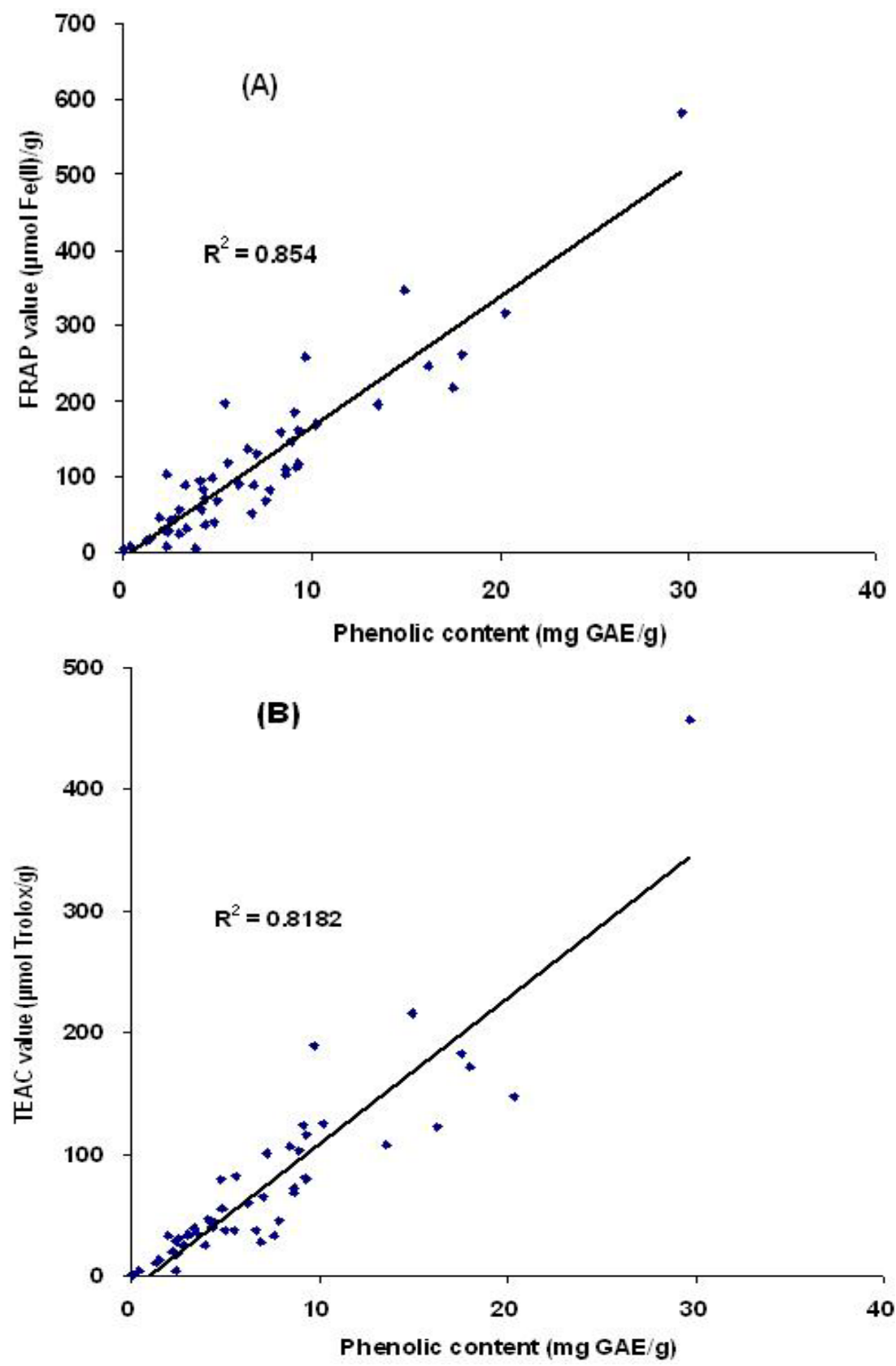

For the correlation between TEAC values and total phenolic contents, the regression equation $\mathrm{Y}=11.968-10.733$ was also statistically significant $(\mathrm{F}=216.107, p<0.001)$, and the coefficient of determination $\mathrm{R}^{2}=0.818$. Figure 2 shows the positive linear correlation, and the total antioxidant capacities of these medicinal plants can be attributed to their phenolic compounds. In the literature, several other studies also found that phenolic compounds were the main contributors of the antioxidant capacities of the medicinal plants tested $[21,25,26]$. However, in some medicinal plants [27] it was also found that there was no significant linear correlation between the antioxidant capacities and the total phenolic contents, which suggested that other compounds, such as polysaccharides, might be the major antioxidant components in those plants [28]. 


\section{Experimental}

\subsection{Chemicals and plant materials}

2,4,6-Tri(2-pyridyl)-s-triazine (TPTZ), 6-hydroxy-2,5,7,8-tetramethylchromane-2-carboxylic acid (Trolox), 2,2'-azinobis(3-ethylbenothiazoline-6-sulfonic acid) diammonium salt (ABTS), FolinCiocalteu's reagent and gallic acid were purchased from Sigma Aldrich (St. Louis, MO, USA). Iron (III) chloride 6-hydrate, iron (II) sulfate 7-hydrate, acetic acid, methanol, hydrochloric acid, acetic acid, sodium acetate, potassium persulphate and sodium carbonate were obtained from Tianjing Chemical Factory (Tianjing, China). All chemicals used in the experiment were of analytical grade. Fifty selected medicinal plants associated with treatment of rheumatic diseases were purchased from Beijing Tong-Ren-Tang drug retail outlet in Guangzhou, China.

\subsection{Sample preparation}

The dry plant samples were ground to a fine powder with a special grinder for herbal medicine. A precisely weighed amount $(0.5 \mathrm{~g})$ of the powder was extracted with $10 \mathrm{ml}$ of $80 \%$ methanol at $35{ }^{\circ} \mathrm{C}$ for $24 \mathrm{~h}$ in a shaking bath according to the literature [12]. All samples were then cooled down to the room temperature and centrifuged at 4,000 rpm for $10 \mathrm{~min}$. The supernatant was recovered for the evaluation of antioxidant capacity and total phenolic content.

\subsection{Ferric-reducing antioxidant power (FRAP) assay}

The FRAP assay was carried out according to the literature [20]. Briefly, the FRAP reagent was prepared from sodium acetate buffer $(300 \mathrm{mM}, \mathrm{pH} 3.6), 10 \mathrm{mM}$ TPTZ solution $(40 \mathrm{mM} \mathrm{HCl}$ as solvent) and $20 \mathrm{mM}$ iron (III) chloride solution in a volume ratio of 10:1:1, respectively. The FRAP reagent was prepared fresh daily and warmed to $37{ }^{\circ} \mathrm{C}$ in a water bath before use. One hundred microliters of the diluted sample was added to $3 \mathrm{~mL}$ of the FRAP reagent. The absorbance of the reaction mixture was then detected at $593 \mathrm{~nm}$ after $4 \mathrm{~min}$ in room temperature. The standard curve was constructed using $\mathrm{FeSO}_{4}$ solution, and the results were expressed as $\mu \mathrm{mol} \mathrm{Fe}(\mathrm{II}) / \mathrm{g}$ dry weight of plant material.

\subsection{Trolox equivalent antioxidant capacity (TEAC) assay}

The TEAC assay was carried out to determine the free radical scavenging capacity using the $\mathrm{ABTS}^{\circ+}$ radical cation, according to the literature [29]. Briefly, the $\mathrm{ABTS}^{\bullet+}$ stock solution was prepared from $7 \mathrm{mM} \mathrm{ABTS}^{\circ+}$ and $2.45 \mathrm{mM}$ potassium persulfate in a volume ratio of $1: 1$, and then should be incubated in the dark for $16 \mathrm{~h}$ at room temperature and used within 2 days. The ABTS ${ }^{\circ+}$ working solution was prepared by dilution of the stock solution with ethanol to an absorbance of $0.70 \pm 0.05$ at $734 \mathrm{~nm}$. All samples were diluted approximately to provide $20-80 \%$ inhibition of the blank absorbance. One hundred microliters of the diluted sample was mixed with $3.8 \mathrm{~mL} \mathrm{ABTS}^{\cdot+}$ working solution. The absorbance of the reaction mixture was then detected at $734 \mathrm{~nm}$ after 6 min of incubation at room temperature, and the percent of inhibition of absorbance at $734 \mathrm{~nm}$ was calculated. 
Trolox solution was used as a reference standard, and the results were expressed as $\mu$ mol Trolox/g dry weight of plant material.

\subsection{Determination of total phenolic content}

The total phenolic contents were determined using Folin-Ciocalteu method according to the literature [23]. Briefly, diluted sample $(0.50 \mathrm{~mL})$ was added to 1:10 diluted Folin-Ciocalteu reagent $(2.5 \mathrm{~mL}$ ). After $4 \mathrm{~min}$, saturated sodium carbonate solution (about $75 \mathrm{~g} / \mathrm{L}, 2 \mathrm{~mL}$ ) was added. After $2 \mathrm{~h}$ of incubation at room temperature, the absorbance of the reaction mixture was measured at $760 \mathrm{~nm}$. Gallic acid was used as a reference standard, and the results were expressed as milligram gallic acid equivalent (mg GAE)/g dry weight of plant material.

\subsection{Statistical analysis}

All the experiments were performed in triplicate, and the results were expressed as mean $\pm \mathrm{SD}$ (standard deviation). The correlation between the antioxidant capacities and total phenolic contents was analyzed using the simple linear regression, and coefficient of determination $\left(\mathrm{R}^{2}\right)$ was calculated. Statistical analysis was performed using SPSS 13.0 and MS Excel 2003. The significant difference was considered at $p$ value $\leq 0.05$.

\section{Conclusions}

The antioxidant capacities and total phenolic contents of 50 selected medicinal plants associated with the treatment of rheumatic diseases were evaluated using the FRAP and TEAC assays as well as the Folin-Ciocalteu method, respectively. Overall, these medicinal plants had relatively high antioxidant capacities and total phenolic contents. A significant correlation between the FRAP values and TEAC values suggested that antioxidants in these plants were capable of reducing oxidants and scavenging free radicals. A positive linear correlation between the antioxidant capacities and total phenolic contents indicated that the phenolic compounds could be the main contributors to the antioxidant capacities of these plants. Several plants (Geranium wilfordii, Loranthus parasiticus, Polygonum aviculare, Pyrrosia sheaeri, Sinomenium acutum and Tripterygium wilfordii) showed the highest antioxidant capacities and total phenolic contents among all the tested species, and they could be potential rich sources of natural antioxidants. Because of their strong antioxidant capacities, these plants are also potential in anti-inflammatory abilities. In the future, the specific compounds with high antioxidant capacities should be isolated, purified and identified from these plants to further develop natural antioxidants and investigate antirheumatic drugs.

\section{Acknowledgements}

This research was supported by the Hundred-Talents Scheme of Sun Yat-sen University. The useful suggestion and technical assistance from Qin Xiao, Meng-Jun Hou and Tie-Jiang Chen is highly appreciated. 


\section{References and Notes}

1. Azizova, O.A. Role of free radical processes in the development of atherosclerosis. Biol. Membrany. 2002, 19, 451-471.

2. Quintero, M.; Brennan, P.A.; Thomas, G.J.; Moncada, S. Nitric oxide is a factor in the stabilization of hypoxia-inducible factor-1 alpha in cancer: Role of free radical formation. Cancer Res. 2006, 66, 770-774.

3. Nagler, R.; Reznick, A.; Shafir, Y.; Shehadeh, N. Free radical-related effects and antioxidants in saliva and serum of adolescents with type 1 diabetes mellitus. Free Radic. Res. 2006, 40, S156.

4. Barnham, K.J.; Masters, C.L.; Bush, A.I. Neurodegenerative diseases and oxidative stress. Nat. Rev. Drug Discov. 2004, 3, 205-214.

5. Wickens, A.P. Ageing and the free radical theory. Resp. Physiol. 2001, 128, 379-391.

6. Stamler, J.S.; Hausladen, A. Oxidative modifications in nitrosative stress. Nat. Struct. Biol. 1998, 5, 247-249.

7. Nordberg, J.; Arner, E.S. Reactive oxygen species, antioxidants and the mammalian thioredoxin system. Free Radic. Biol. Med. 2001, 31, 1287-1312.

8. Duthie, G.G.; Duthie, S.J.; Kyle, J.A.M. Plant polyphenols in cancer and heart disease: Implications as nutritional antioxidants. Nutr. Res. Rev. 2000, 13, 79-106.

9. Li, H.B.; Li, D.; Zhang, Y.; Gan, R.Y.; Song, F.L.; Chen, F. Antioxidant properties of Chinese medicinal plants. In Reactive Oxygen Species and Antioxidants in Higher Plants; Gupta, S.D., Ed.; Sciences Publishers: Enfield, CT, USA, 2010; Chapter 15, pp. 331-362.

10. Wong, C.C.; Li, H.B.; Cheng, K.W.; Chen, F. A systematic survey of antioxidant activity of 30 Chinese medicinal plants using the ferric reducing antioxidant power assay. Food Chem. 2006, 97, 705-711.

11. Liu, H.Y.; Qiu, N.X.; Ding, H.H.; Yao, R.Q. Polyphenols contents and antioxidant capacity of 68 Chinese herbals suitable for medical or food uses. Food Res. Int. 2008, 41, 363-370.

12. Cai, Y.Z.; Luo, Q.; Sun, M.; Corke, H. Antioxidant activity and phenolic compounds of 112 traditional Chinese medicinal plants associated with anticancer. Life Sci. 2004, 74, 2157-2184.

13. Chen, K.L.; Plumb, G.W.; Bennett, R.N.; Bao, Y.P. Antioxidant activities of extracts from five anti-viral medicinal plants. J. Ethnopharmacol. 2005, 96, 201-205.

14. Li, H.B.; Wong, C.C.; Cheng, K.W.; Chen, F. Antioxidant properties in vitro and total phenolic contents in methanol extracts from medicinal plants. LWT-Food Sci. Technol. 2008, 41, 385-390.

15. Zhu, Y.Z.; Huang, S.H.; Tan, B.K.H.; Sun, J.; Whiteman, M.; Zhu, Y.C. Antioxidants in Chinese herbal medicines: A biochemical perspective. Nat. Prod. Rep. 2004, 21, 478-489.

16. Liao, H.; Banbury, L.K.; Leach, D.N. Antioxidant activity of 45 Chinese herbs and the relationship with their TCM characteristics. Evid. Bas. Complem. Alternat. Med. 2008, 5, 429-434.

17. Dragland, S.; Senoo, H.; Wake, K.; Holte, K.; Blomhoff, R. Several culinary and medicinal herbs are important sources of dietary antioxidants. J. Nutr. 2003, 133, 1286-1290.

18. Delfino, R.J.; Staimer, N.; Tjoa, T.; Polidori, A.; Arhami, M.; Gillen, D.L.; Kleinman, M.T.; Vaziri, N.D.; Longhurst, J.; Zaldivar, F.; Siouta, S.C. Circulating biomarkers of inflammation, 
antioxidant activity and platelet activation are associated with primary combustion aerosols in subjects with coronary artery disease. Environ. Health Persp. 2008, 116, 898-906.

19. Schinella, G.R.; Tournier, H.A.; Prieto, J.M.; Mordujovich de Buschiazzo, P.; Ríos J.L. Antioxidant activity of anti-inflammatory plant extracts. Life Sci. 2002, 70, 1023-1033.

20. Benzie, I.F.; Strain, J.J. The ferric reducing ability of plasma (FRAP) as a measure of "antioxidant power": The FRAP assay. Anal. Biochem. 1996, 239, 70-76.

21. Katalinic, V.; Milos, M.; Kulisic, T.; Jukic, M. Screening of 70 medicinal plant extracts for antioxidant capacity and total phenols. Food Chem. 2006, 94, 550-557.

22. Li, H.B.; Cheng, K.W.; Wong, C.C.; Fan, K.W.; Chen, F.; Jiang, Y. Evaluation of antioxidant capacity and total phenolic content of different fractions of selected microalgae. Food Chem. 2007, 102, 771-776.

23. Singleton, V.L.; Orthofer, R.; Lamuela-Raventos, R.M. Analysis of total phenols and other oxidation substrates and antioxidants by means of Folin-Ciocalteu reagent. Method Enzymol. 1999, 299, 152-178.

24. Djeridane, A.; Yousfi, M.; Nadjemi, B.; Boutassouna, D.; Stocker, P.; Vidal, N. Antioxidant activity of some Algerian medicinal plants extracts containing phenolic compounds. Food Chem. 2006, 97, 654-660.

25. Zheng, W.; Wang, S.Y. Antioxidant activity and phenolic compounds in selected herbs. J. Agric. Food Chem. 2001, 49, 5165-5170.

26. Tawaha, K.; Alali, F.Q.; Gharaibeh, M.; Mohammad, M.; El-Elimat, T. Antioxidant activity and total phenolic content of selected Jordanian plant species. Food Chem. 2007, 104, 1372-1378.

27. Hinneburg, I.; Dorman, H.J.D.; Hiltunen, R. Antioxidant activities of extracts from selected culinary herbs and spices. Food Chem. 2006, 97, 122-129.

28. Kardosova, A.; Machova, E. Antioxidant activity of medicinal plant polysaccharides. Fitoterapia 2006, 77, 367-373.

29. Re, R.; Pellegrini, N.; Proteggente, A.; Pannala, A.; Yang, M.; Rice-Evans, C. Antioxidant activity applying an improved ABTS radical cation decolorization assay. Free Radic. Biol. Med. 1999, 26, 1231-1237.

Sample Availability: Plant samples are available from the authors.

(C) 2010 by the authors; licensee MDPI, Basel, Switzerland. This article is an Open Access article distributed under the terms and conditions of the Creative Commons Attribution license (http://creativecommons.org/licenses/by/3.0/). 